\title{
Use of Technology-Based Tools to Support Adolescents and Young Adults With Chronic Disease: Systematic Review and Meta-Analysis
}

Jac Kee Low, BSc (Hons), PhD; Elizabeth Manias, RN, PhD, MN, MPharm

School of Nursing and Midwifery, Centre for Quality and Patient Safety Research, Deakin University, Burwood, Australia

\section{Corresponding Author:}

Jac Kee Low, BSc (Hons), PhD

School of Nursing and Midwifery

Centre for Quality and Patient Safety Research

Deakin University

Burwood,

Australia

Phone: 61392446729

Email: jac.low@deakin.edu.au

\section{Abstract}

Background: With the large amount of material that is readily available on the internet, there are endless opportunities for electronic health-literate patients to obtain and learn new information. Although novel, a Web- or mobile-based program can be a powerful way to engage adolescents and young adults (AYAs). The ongoing engagement of AYAs with chronic disease is vital not only to empower them but also to ensure a smooth transition from pediatric to adult health care.

Objective: This study aimed to evaluate the current evidence on Web- or mobile-based interventions designed for AYAs.

Methods: This review was registered with PROSPERO: CRD42018096487. A systematic search of MEDLINE Complete, EMBASE, and CINAHL Complete was conducted on April 10, 2019, for studies that examined the perspectives of transition-age patients about technology-based interventions, the process involved in intervention development, or the evaluation of intervention efficacy. For each study, the comprehensiveness of reporting was appraised. The Downs and Black checklist was used for intervention efficacy trials, the Standards for Reporting Qualitative Research checklist was used for qualitative work, and a 16-item tool developed by Tong et al was used for questionnaire research.

Results: The search uncovered 29 relevant studies, which included qualitative studies $(n=14)$, intervention efficacy studies $(n=7)$, questionnaire studies $(n=4)$, mixed qualitative and questionnaire studies $(n=2)$, and a mixed qualitative and pilot randomized controlled trial study $(\mathrm{n}=1)$. The reporting comprehensiveness score of questionnaires was rated considerably lower $(\mathrm{n}=6,13 \%-57 \%$ [2/16-8/14]) than the scores of intervention efficacy trials $(\mathrm{n}=8,48 \%-85 \%$ [13/27-23/27]) and qualitative research $(\mathrm{n}=17,40 \%-93 \%$ [8.5/21-19.5/21]). AYAs were receptive to obtaining information via a website or mobile app. An intervention was more likely to be perceived as useful by AYAs when there was a concerted effort to involve AYAs and subject matter experts in the process of intervention design, as opposed to relying solely on the AYAs or the experts alone. The preferred medium of intervention delivery varied greatly for AYAs, ranging from static text to audiovisual materials. However, AYAs considered being concise was the most important aspect. Across different conditions, AYAs were interested in receiving information on diverse topics, such as anxiety and stress management, dealing with insurance, and having social relationships. Patients also requested for disease-specific information, such as weather forecasts and pollen levels for patients with asthma and information related to the pretransplant period for organ transplant recipients. Meta-analyses showed no significant group differences across time on quality of life, self-efficacy, and self-management.

Conclusions: Owing to the lack of intervention efficacy trials, no conclusion can be drawn if an intervention delivered via a mobile app is better than that delivered via a website. However, through this systematic review, it is confirmed that AYAs were receptive to receiving medical information electronically.

(JMIR Mhealth Uhealth 2019;7(7):e12042) doi: 10.2196/12042

\section{KEYWORDS}

young adult; adolescent; self-management; transition to adult care; disease management; systematic review 


\section{Introduction}

\section{Background}

The number of children living with a chronic disease has grown over the past few decades [1-4]. For example, the incidence rate of childhood type 1 diabetes continues to rise by approximately $3 \%$ per annum [1,2], whereas the prevalence of cancer increases at $0.6 \%$ per annum [4]. The increase in incidence and prevalence may be attributable to medical advances that improve screening and diagnosis in addition to disease management, which altogether offer a better chance of patient survival. One such example is sickle cell disease, which had very few children surviving into adulthood in the 1970s, but $95 \%$ of those born in the recent decade will reach their 18th birthday [5]. The change in health care need is inevitable as medical advances are made. To receive age-appropriate care, young patients with pediatric-onset chronic disease need to transition from pediatric to adult health care. Transition is defined as the planned process of preparing adolescents and young adults (AYAs) as they move from caregiver-directed care in a pediatric unit to disease self-management in an adult unit [6].

Although AYAs are no longer children, they are yet to identify themselves as adults. They may be reluctant to detach from the pediatric unit, they may not feel comfortable in the adult care environment, and they may be fearful of their own future after confronting older and sicker patients in the adult unit [7]. If they are not prepared adequately, the transfer to an adult care environment can be problematic, which could cause clinic nonattendance and treatment nonadherence [8-14]. Evidence shows that outpatient clinic attendance among AYAs with chronic diseases, such as type 1 diabetes and sickle cell disease, declines significantly when comparing the pretransfer period with the posttransfer period [8-10]. A study conducted in the United Kingdom reported that $98 \%$ of 229 young people with diabetes attended a clinic appointment at least 6-monthly 2 years pretransfer but the proportion declined to $61 \%$ at 2 years posttransfer [11]. In a 2015 systematic review, Heery et al [12] reported that between $28 \%$ and $63 \%$ of adults with congenital heart disease had $\geq 2$ years lapse in care after leaving the pediatric care in Canada, the United Kingdom, and the United States. In a retrospective study involving liver transplant recipients, immunosuppressive medication adherence significantly decreased over time from pretransfer to 2 years posttransfer [14]. These are worrying trends. Not only does patient nonadherence exacerbate symptoms and cause disease progression, but it also leads to the eventual need for more intensive monitoring and expensive treatment. However, there is evidence that an age-appropriate transition program can improve patient outcomes, which include attendance rates and medication adherence in adolescents with inflammatory bowel disease [13] and disease-specific knowledge and satisfaction with care in those with juvenile arthritis [15]. Hence, how the process of transition is managed plays a crucial role in the ongoing engagement of AYAs with the health care system.

To facilitate a successful transition, AYAs with chronic disease need to be equipped with self-management skills and be engaged with their treatment plan to maintain positive health outcomes
[16]. A Cochrane review by Campbell et al [17], which was conducted in 2016, only found 4 small randomized controlled trials with sample sizes that ranged from 26 to 81 . The trials covered a limited range of interventions, which included a 2-day face-to-face-delivered workshop [18], an 8-month Web-based and text-delivered disease management and skill-based intervention [19], a one-off meeting with a nurse [20], and a structured transition program involving a transition coordinator over a 12-month period [21]. No firm conclusions could be drawn from the intervention studies [17].

\section{Objective}

An emerging area that is worth exploring is the use of Web- or mobile-based materials to engage AYAs. It can be an innovative way to build their skills and prepare them for the transition process [22,23]. As parental and clinician assumptions may fall short of identifying the needs of AYA patients, it is critical to obtain knowledge of AYAs' perspectives. This knowledge will help ensure that the needs of AYA patients are addressed and that AYAs are appropriately supported through the designed intervention during their transition from pediatric to adult health care. This systematic review aimed to evaluate the current evidence on Web- or mobile-based interventions by summarizing studies that examined either the perspectives of AYAs or intervention efficacy.

\section{Methods}

\section{Protocol and Registration}

The protocol for this systematic review was registered with PROSPERO 2018: CRD42018096487 [24]. It was conducted in accordance with the Preferred Reporting Items for Systematic Reviews and Meta-Analyses guidelines (PRISMA) [25].

\section{Eligibility Criteria}

Studies that met the following criteria were included in the review:

- There were no restrictions on the study design, provided that it was primary research exploring the perspectives of patients about a technology-based intervention; a methods paper describing the intervention development process; or primary research evaluating intervention effectiveness.

- The intervention must be freely available through a device that can be connected to the internet in the form of an app on a mobile device or on the World Wide Web; designed for patients with at least one chronic disease; and accessible by patients at any time.

- $\quad$ Study participants could be aged less than 18 years or adults (aged $\geq 18$ years) who were either transitioning or had already transitioned to adult health care services.

Studies were excluded based on the following criteria:

- The only aim of the technology component of the intervention was to allow participants to engage with another party online, such as forums and social media platforms; test a serious game; test an equipment, such as a Bluetooth spirometer and blood glucose monitors; and 
remotely monitor patient progress, such as patient portals and symptom reporting platform.

- Studies where pediatric group findings could not be delineated from research involving other patient groups, such as middle-aged persons and older patients.

\section{Search Strategy}

Overall, 3 electronic databases were searched: MEDLINE Complete via EBSCOhost (1967 to March 31, 2019), EMBASE (1972 to March 31, 2019), and CINAHL Complete via EBSCOhost (1978 to March 31, 2019). The search utilized terms associated with the concepts of technology, transition or disease management, chronic disease, and adolescents or young adults. An example of the search strategy is included in Multimedia Appendix 1.

\section{Study Selection}

Search results were collated in a reference manager (EndNote X8, Clarivate Analytics, 2017), duplicates were deleted, and the results were exported to a spreadsheet (Microsoft Excel, Microsoft Corporation, 2016) for initial screening of titles and abstracts. The screening was conducted independently by 2 reviewers (JKL and EM) whereby a priori inclusion and exclusion criteria were applied. The same reviewers further reviewed the full texts of articles independently to select studies for inclusion according to the eligibility criteria. Manual checks on the reference lists of retrieved reviews on the relevant topic were conducted to identify articles not found by the database searches. Discordance between reviewers was resolved through discussion.

\section{Data Extraction and Quality Assessment}

For each included study, the following items were extracted: study characteristics, participant demographics, and study design using a standardized form entered into Microsoft Excel. Data related to the design of a technology-based intervention were also extracted.
For studies using multiple methods, the comprehensiveness of reporting was appraised using checklists applicable to the major methodological approach of each study. The Downs and Black checklist (D\&B) was used for intervention efficacy trials [26], the Standards for Reporting Qualitative Research (SRQR) checklist was used for qualitative research [27], and a 16-item tool developed by Tong et al [28] was used for questionnaire research. Owing to the lack of clarity on how to score item 27 in the D\&B checklist (power calculation), a score of 0 or 1 was allocated to indicate whether the authors achieved their target sample size or not. If no power calculation was conducted a priori, a score of 0 was given for item 27 . The same approach has been used by previous researchers $[29,30]$. No study was excluded based on the quality assessment.

\section{Data Synthesis and Analysis}

Data collected from all qualitative studies, including mixed-methods, exploratory, and feasibility studies, using interviews and focus groups in addition to open-ended responses to items in questionnaires, were extracted and organized using NVivo (version 11, QSR International Pty Ltd). Qualitative data were pooled and thematically analyzed using the method outlined by Thomas and Harden [31] by a reviewer (JKL). Quantitative data were pooled for meta-analyses using RevMan (version 5.3, The Cochrane Collaboration). Where a meta-analysis could not be conducted as a result of the small number of available studies, a descriptive synthesis of the quantitative findings was undertaken instead.

\section{Results}

\section{Study Selection}

The initial search yielded 235 records. Of these, 89 were selected for full-text review, which led to further exclusion of 60 studies because of ineligibility (Figure 1). Overall, 29 studies were included in the review [19,32-59]. 
Figure 1. Study flow diagram.

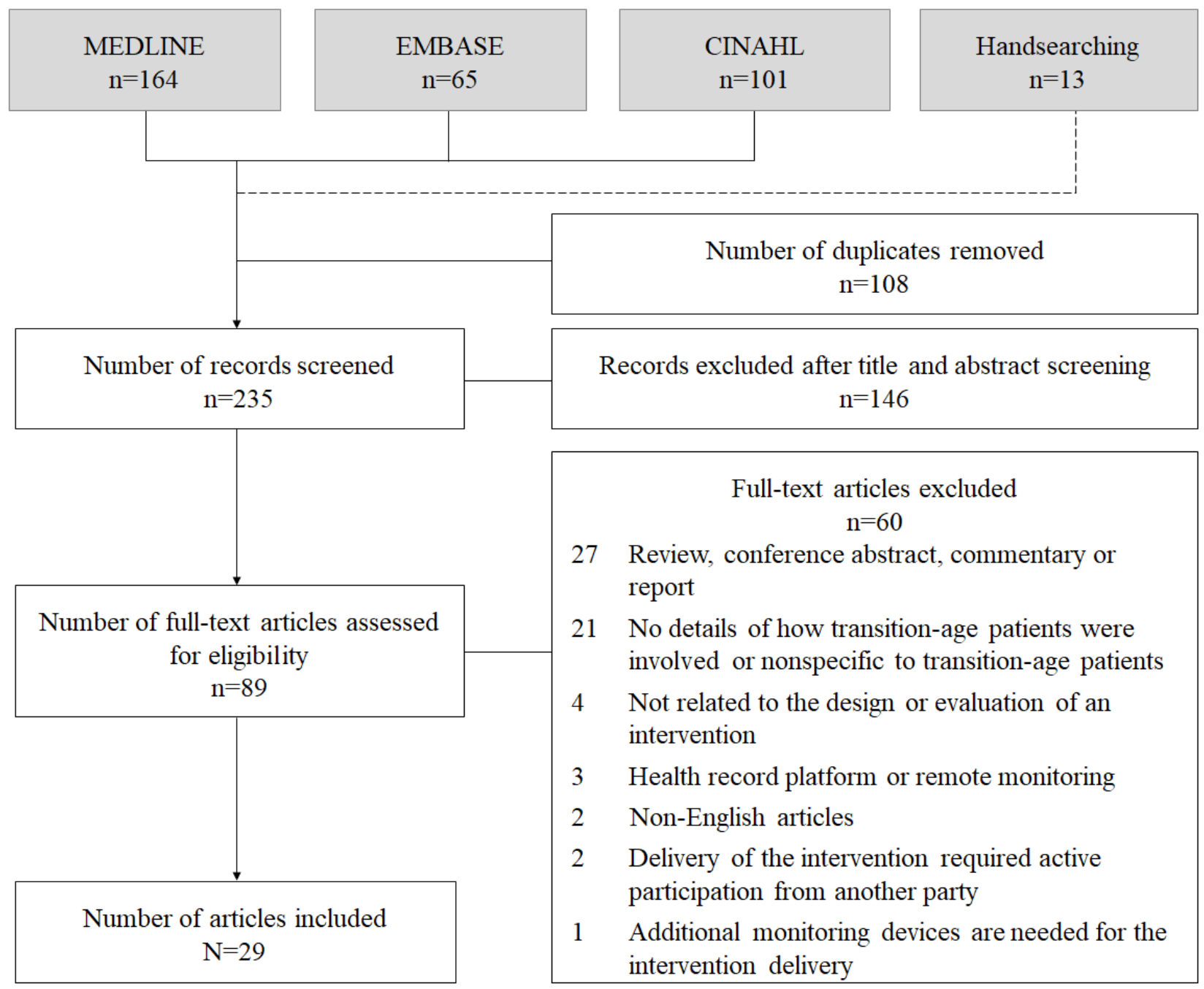

\section{Study Characteristics ( $\mathrm{N}=29)$}

All studies $(\mathrm{N}=29)$ were conducted in developed countries (Table 1). Diabetes was the most investigated type of chronic disease $(n=9)$ followed by rheumatic disease $(n=6)$, asthma $(n=5)$, and cystic fibrosis $(n=4)$, whereas 2 studies did not specify the type of chronic disease examined [32,35]. Participants were aged between 7 and 28 years (Table 2).

A total of 17 studies utilized a qualitative approach: 6 exploratory studies [32,39,51,53,54,57], 3 multiphase studies involving the iterative design and development of an intervention [43,47,52], 2 feasibility studies [34,45], 2 usability studies of an intervention [42,55], 1 evaluation of user experience [50], and 3 mixed-methods studies [35,38,58].

Among the included studies, 6 studies had a questionnaire component in the study design: a feasibility study [33], a cross-sectional survey to inform the development of an eHealth intervention [40], a multiphase study involving the iterative development and user evaluation of an intervention [36], an evaluation of user engagement [59], and 2 mixed-methods studies [35,38].

In addition, 8 studies included an intervention efficacy trial, of which one was a mixed-methods study [58]. The conduct of a randomized controlled trial was the most popular design $(n=3)$ $[19,41,44]$ followed by a pilot randomized controlled trial $(n=3)$ $[37,56,58]$. The remaining intervention efficacy trials utilized a nonrandomized controlled study design [48] and a pretest -posttest design [46]. 
Table 1. Description of included studies ( $\mathrm{N}=29)$.

\begin{tabular}{|c|c|c|c|c|}
\hline Study identity & Country & Study design & Type of chronic disease & Quality assessment \\
\hline Abraham et al [32] & United States & $\begin{array}{l}\text { Exploratory qualitative design using } \\
\text { semistructured interview }\end{array}$ & $-^{\mathrm{a}}$ & $\mathrm{SRQR}^{\mathrm{b}}: 15.5 / 21$ \\
\hline Ammerlaan et al [33] & Netherlands & $\begin{array}{l}\text { Quantitative feasibility study using a Web- } \\
\text { based questionnaire }\end{array}$ & Rheumatic disease & Tong $^{c}:$ 7/13 \\
\hline Ammerlaan et al [34] & Netherlands & $\begin{array}{l}\text { Qualitative feasibility study using } \\
\text { semistructured interview }\end{array}$ & Juvenile idiopathic arthritis & SRQR: 17/21 \\
\hline Applebaum et al [35] & United States & $\begin{array}{l}\text { Mixed methodologies, cross-sectional study: } \\
\text { questionnaire and qualitative using focus } \\
\text { group }\end{array}$ & - & Tong: 8/14; SRQR: 9/21 \\
\hline Ashurst et al [36] & United Kingdom & $\begin{array}{l}\text { 2-stage approach: stage } 1 \text { development and } \\
\text { stage } 2 \text { evaluation using email and a Web- } \\
\text { based questionnaire }\end{array}$ & Diabetes & Stage 2 Tong: $6 / 16$ \\
\hline Breakey et al [37] & Canada & Pilot randomized control trial & Hemophilia & $D \& B^{d}: 19 / 27$ \\
\hline Coyne et al [38] & Ireland & $\begin{array}{l}\text { Four-phase participatory iterative approach } \\
\text { using questionnaire, one-to-one interview, } \\
\text { participatory workshop, and Google Analyt- } \\
\text { ics }\end{array}$ & $\begin{array}{l}\text { Diabetes, cystic fibrosis, or } \\
\text { congenital heart disease }\end{array}$ & $\begin{array}{l}\text { Phase } 1 \text { Tong: } 2 / 14 \text {; Phase } \\
1 \text { SRQR: } 11 / 21\end{array}$ \\
\hline Huang et al [39] & United States & $\begin{array}{l}\text { Exploratory qualitative design using a focus } \\
\text { group }\end{array}$ & $\begin{array}{l}\text { Diabetes, cystic fibrosis, or } \\
\mathrm{IBD}^{\mathrm{e}}\end{array}$ & SRQR: $15 / 21$ \\
\hline Huang et al [19] & United States & Randomized controlled trial & $\begin{array}{l}\text { Diabetes, cystic fibrosis, or } \\
\text { IBD }\end{array}$ & D\&B: $23 / 27$ \\
\hline Johnson et al [40] & United States & $\begin{array}{l}\text { Cross-sectional study: Web-based question- } \\
\text { naire }\end{array}$ & Juvenile arthritis & Tong: $7 / 14$ \\
\hline Joseph et al [41] & United States & Randomized controlled trial & Asthma symptoms & D\&B: $19 / 27$ \\
\hline Korus et al [42] & Canada & $\begin{array}{l}\text { Qualitative usability testing approach using } \\
\text { semistructured interview }\end{array}$ & $\begin{array}{l}\text { Solid organ transplant recip- } \\
\text { ient }\end{array}$ & SRQR: $15 / 21$ \\
\hline Lopez et al [43] & United States & $\begin{array}{l}\text { Formative iterative process using } \\
\text { semistructured interview and group inter- } \\
\text { view }\end{array}$ & Congenital heart disease & SRQR: 17/21 \\
\hline Mulvaney et al [44] & United States & Randomized controlled trial & Diabetes & D\&B: $15 / 27$ \\
\hline Mulvaney et al [45] & United States & Qualitative feasibility study & Diabetes & SRQR: 11.5/21 \\
\hline Paul [46] & Australia & $\begin{array}{l}\text { Two-phase, multimethod approach: phase } \\
1 \text { evaluation of intervention fidelity and } \\
\text { phase } 2 \text { feasibility pre- to posttest }\end{array}$ & Diabetes & Phase 2 D\&B: $14 / 27$ \\
\hline Peters et al [47] & Australia & $\begin{array}{l}\text { Multiphase, participatory user research } \\
\text { study using participatory workshop, work- } \\
\text { book, and user evaluation }\end{array}$ & Asthma & SRQR: 16/21 \\
\hline Runge et al [48] & Germany & Nonrandomized trial & Asthma & D\&B: $17 / 27$ \\
\hline $\begin{array}{l}\text { Scal et al [49], Secor- } \\
\text { Turner et al [60] }\end{array}$ & United States & $\begin{array}{l}\text { Descriptive paper on the intervention devel- } \\
\text { opment process }\end{array}$ & Juvenile arthritis & $\mathrm{N} / \mathrm{A}^{\mathrm{f}}$ \\
\hline Schneider et al [50] & United States & $\begin{array}{l}\text { Qualitative evaluation of user experience } \\
\text { using semistructured interview }\end{array}$ & Asthma & SRQR: 14/21 \\
\hline Schneider et al [51] & United States & $\begin{array}{l}\text { Exploratory qualitative design using } \\
\text { semistructured interview }\end{array}$ & Asthma & SRQR: 18/21 \\
\hline Simmons et al [52] & United States & $\begin{array}{l}\text { Multiphase, iterative design and develop- } \\
\text { ment of an intervention: environmental } \\
\text { scan, Web-based or telephone focus group, } \\
\text { and in-person focus group }\end{array}$ & Hemophilia & SRQR: 13.5/21 \\
\hline Slater et al [53] & Australia & $\begin{array}{l}\text { Exploratory qualitative design using } \\
\text { semistructured interview and focus group }\end{array}$ & Musculoskeletal pain & SRQR: 19/21 \\
\hline Sterling et al [54] & Canada & $\begin{array}{l}\text { Exploratory qualitative design using } \\
\text { semistructured interview }\end{array}$ & Hemophilia & SRQR: 15/21 \\
\hline
\end{tabular}




\begin{tabular}{|c|c|c|c|c|}
\hline Study identity & Country & Study design & Type of chronic disease & Quality assessment \\
\hline Stinson et al [55] & Canada & $\begin{array}{l}\text { Qualitative usability testing with } \\
\text { semistructured interview }\end{array}$ & Juvenile idiopathic arthritis & SRQR: $18.5 / 21$ \\
\hline Stinson et al [56] & Canada & Pilot randomized controlled trial & Juvenile idiopathic arthritis & D\&B: $21 / 27$ \\
\hline Stinson et al [57] & Canada & $\begin{array}{l}\text { Descriptive exploratory qualitative design } \\
\text { using focus group and interview }\end{array}$ & Chronic pain & SRQR: 19.5/21 \\
\hline Whittemore et al [58] & United States & $\begin{array}{l}\text { Multiphase mixed-methods design: qualita- } \\
\text { tive using focus group and think-aloud } \\
\text { method, followed by a feasibility and pilot } \\
\text { study }\end{array}$ & Diabetes & $\begin{array}{l}\text { Phase 1 SRQR: } 8.5 / 21 \text {; } \\
\text { phase } 2 \text { D\&B: } 13 / 27\end{array}$ \\
\hline Zhao et al [59] & Australia & Pilot study using questionnaire & $\begin{array}{l}\text { Diabetes, cystic fibrosis, or } \\
\text { IBD }\end{array}$ & Tong: $2 / 16$ \\
\hline
\end{tabular}

${ }^{\mathrm{a}}$ Not specified or not reported.

${ }^{b}$ SRQR: Standards for Reporting Qualitative Research.

${ }^{\mathrm{c}}$ Tong: the 16-item checklist questionnaire developed by Tong et al [28].

${ }^{\mathrm{d} D \& B}$ : Downs and Black checklist for intervention efficacy trial.

${ }^{\mathrm{e}}$ IBD: inflammatory bowel disease.

${ }^{\mathrm{f}} \mathrm{N} / \mathrm{A}$ : not applicable. 
Table 2. Description of participant demographics.

\begin{tabular}{|c|c|c|c|c|}
\hline Study identity & Number of participants ${ }^{\mathrm{a}}$ & Mean age $(\mathrm{SD} \text { or range })^{\mathrm{a}}$ & Age group $(n)^{\mathrm{a}}$ & Gender (male), $\mathrm{n}(\%)^{\mathrm{a}}$ \\
\hline Abraham et al [32] & 20 & $-^{b}$ & $\begin{array}{l}7-11(4), 12-14(9), 15-17 \\
(7)\end{array}$ & $8(40)$ \\
\hline Ammerlaan et al [33] & $\mathrm{IG}^{\mathrm{c}}: 10 ; \mathrm{CG}^{\mathrm{d}}: 9$ & IG: 22.3 (17-25); CG: 20.7 (17-25) & - & IG: 1 (10); CG: (2) 22 \\
\hline Ammerlaan et al [34] & 13 & $20(17-22)$ & - & $1(8)$ \\
\hline Applebaum et al [35] & $\begin{array}{l}\text { Questionnaire: } 35 \text {; Focus } \\
\text { group: } 20\end{array}$ & $\begin{array}{l}\text { Questionnaire: } 16.9 \text { (13-20); Focus } \\
\text { group: - }\end{array}$ & - & Survey: 9 (26); Focus group: \\
\hline Ashurst et al [36] & Stage 1: 6; Stage 2: 83 & $\begin{array}{l}\text { Stage 1: } 20.3 \text { (3.3); Stage 2: } 19.0 \\
(2.6)\end{array}$ & - & Stage 1: —; Stage 2: 37 (45) \\
\hline Breakey et al [37] & 29 & IG: 16.0 (1.4); CG: 16.1 (1.4) & - & $29(100)$ \\
\hline Coyne et al [38] & $\begin{array}{l}\text { Phase } 1 \text { questionnaire: } \\
207 \text {, interview: } 21 \text {; phase } \\
2 \text { co-design group: } 5 \text {, } \\
\text { telephone interview: } 4 \text {, } \\
\text { participatory workshop: } \\
12\end{array}$ & - & $\begin{array}{l}\text { Phase } 1 \text { questionnaire: } 14- \\
25 \text { (207), interview partici- } \\
\text { pants: } 14-25 \text { (21); phase } 2 \\
\text { co-design group: } 15-25(5) \text {, } \\
\text { telephone interview: } 15-25 \\
\text { (4), participatory workshop: } \\
\text { 15-25 (12) }\end{array}$ & $\begin{array}{l}\text { Phase } 1 \text { questionnaire: - } \\
\text { interview participants: —; } \\
\text { phase } 2 \text { co-design group: } 2 \\
\text { (40), telephone interview: } \\
\text { _, participatory workshop: }\end{array}$ \\
\hline Huang et al [39] & 10 & $20(18-25)$ & - & $4(40)$ \\
\hline Huang et al [19] & IG: 40; CG: 41 & IG: $17(12-20)^{\mathrm{e}} ; \mathrm{CG}: 17(12-19)^{\mathrm{e}}$ & - & IG: 17 (43); CG: 20 (49) \\
\hline Johnson et al [40] & 134 & $\begin{array}{l}\text { High PedsQL_Psycho: } 15.9(-) \\
\text { low PedsQL_Psycho: } 16.3(-)\end{array}$ & - & $\begin{array}{l}\text { High PedsQL_Psycho: } 10 \\
\text { (15); low PedsQL_Psycho: } \\
12 \text { (18) }\end{array}$ \\
\hline Joseph et al [41] & 314 & $15.3(1.0)$ & - & $115(37)$ \\
\hline Korus et al [42] & 21 & - & $12-14(7), 15-17(13), 18(1)$ & $14(67)$ \\
\hline Lopez et al [43] & Phase 2 expert panel: 6 & Phase 2 expert panel: $16(15-19)^{\mathrm{e}}$ & - & Phase 2 expert panel: 2 (33) \\
\hline Mulvaney et al [44] & IG: 48; CG: 24 & IG: 15.1 (1.5); CG: 15.1 (1.3) & - & IG: 25 (52); CG: 15 (62) \\
\hline Mulvaney et al [45] & 41 & IG only: $15.1(1.5)$ & - & IG only: $21(51)$ \\
\hline Paul [46] & $\begin{array}{l}\text { Phase } 1:- \text {; phase } 2: 5 \\
\text { (Only } 3 \text { participants } \\
\text { completed phase } 2 \text { ) }\end{array}$ & - & $\begin{array}{l}\text { Phase } 1:- \text {; phase } 2: 14(1), \\
15(3), 17(1)\end{array}$ & Phase 1: 一; phase 2: 3 (60) \\
\hline Peters et al [47] & 20 & $17.8(15-24)$ & - & $8(40)$ \\
\hline Runge et al [48] & 178 & $\begin{array}{l}\text { IG1: } 11.1 \text { (2.4); IG2: } 11.0 \text { (2.2); CG: } \\
11.5 \text { (2.9) }\end{array}$ & - & $\begin{array}{l}\text { IG1: } 47 \text { (55); IG2: } 29 \text { (66); } \\
\text { CG: } 38 \text { (79) }\end{array}$ \\
\hline $\begin{array}{l}\text { Scal et al [49], Secor- } \\
\text { Turner et al [60] }\end{array}$ & $\begin{array}{l}\text { Youth: 5; Young adults: } \\
5\end{array}$ & $\begin{array}{l}\text { Youth:16.2 (14-21) }{ }^{\mathrm{f}} \text {; Young adults: } \\
25.4(22-28)^{\mathrm{f}}\end{array}$ & - & $\begin{array}{l}\text { Youth: } 2(40)^{\mathrm{f}} \text {; young } \\
\text { adults: } 1(20)^{\mathrm{f}}\end{array}$ \\
\hline Schneider et al [50] & 16 & - & $13-18(16)$ & - \\
\hline Schneider et al [51] & 20 & $14.4(1.6)$ & - & $9(45)$ \\
\hline Simmons et al [52] & $\begin{array}{l}\text { Web-based focus group: } \\
\text { 40; In-person message } \\
\text { testing focus group: } 19\end{array}$ & - & $\begin{array}{l}\text { Web-based focus group: 16- } \\
17 \text { (24), 18-19 years (16); } \\
\text { In-person message testing } \\
\text { focus group: } 16-17 \text { (12), 18- } \\
19 \text { (7) }\end{array}$ & - \\
\hline Slater et al [53] & 23 & $20.8(2.4)$ & - & $3(13)$ \\
\hline Sterling et al [54] & 11 & $16.3(12.8-18.3)$ & - & $11(100)$ \\
\hline Stinson et al [55] & 19 & $15.7(1.5)$ & - & $5(26)$ \\
\hline Stinson et al [56] & IG: 22; CG: 24 & IG: 14.4 (1.3); CG: 14.8 (1.7) & - & IG: 7 (32); CG: 8 (33) \\
\hline Stinson et al [57] & 23 & - & $14-18(23)$ & $5(22)$ \\
\hline
\end{tabular}




\begin{tabular}{|c|c|c|c|c|}
\hline Study identity & Number of participants ${ }^{\mathrm{a}}$ & Mean age (SD or range) $)^{\mathrm{a}}$ & Age group $(n)^{\mathrm{a}}$ & Gender (male), $\mathrm{n}(\%)^{\mathrm{a}}$ \\
\hline Whittemore et al [58] & $\begin{array}{l}\text { Phase } 1 \text { intervention de- } \\
\text { velopment: } 3 \text {; Phase } 2 \\
\text { randomized pilot trial: } \\
12 \text {; program evaluation: } \\
10\end{array}$ & $\begin{array}{l}\text { Phase } 1 \text { intervention development: } \\
\text {-; phase } 2 \text { randomized pilot trial: } \\
14.4(0.9) \text {; program evaluation: } 14.0 \\
\text { (1.2) }\end{array}$ & - & $\begin{array}{l}\text { Phase } 1 \text { intervention devel- } \\
\text { opment: -; phase } 2 \text { random- } \\
\text { ized pilot trial: } 5 \text { (42); Pro- } \\
\text { gram evaluation: } 6 \text { (60) }\end{array}$ \\
\hline Zhao et al [59] & 10 & $20.2(-)$ & - & $3(30)$ \\
\hline
\end{tabular}

${ }^{\mathrm{a}}$ Characteristics of parent, health care professional, or healthy participants are not included.

${ }^{\mathrm{b}}$ Not specified or not reported.

${ }^{\mathrm{c}} \mathrm{IG}$ : intervention group.

${ }^{\mathrm{d}} \mathrm{CG}$ : comparison group.

${ }^{\mathrm{e}}$ Median years (minimum-maximum).

f Information was obtained from a related, secondary source.

\section{Comprehensiveness of Reporting}

An evaluation of the comprehensiveness of reporting was conducted for all studies, except for Scal et al [49] as the authors did not present any qualitative or quantitative data, and it merely provided a description of the intervention development process. All mixed-methods studies were assessed using 2 separate checklists $[35,38,58]$. One study, which was identified by the authors as a mixed-methods approach, was actually a study involving multiple methods including a literature review, Web-based or telephone focus group, and in-person focus group [52]. Overall, the reporting of questionnaires was rated considerably lower $(n=6$, scores from the questionnaire developed by Tong et al [28]: $13 \%-57 \%$ [2/16-8/14]) than the intervention efficacy trials $(\mathrm{n}=8, \mathrm{D} \& \mathrm{~B}$ scores: $48 \%-85 \%$ [13/27-23/27]) and qualitative research $(n=17$, SRQR scores: $40 \%-93 \%$ [8.5/21-19.5/21]).

Of the 17 qualitative research papers, 4 were missing at least $40 \%$ of the items considered important on the SRQR checklist (Multimedia Appendix 2). Most studies were lacking in detail on the data processing of participants' responses before data analysis, such as the procedure to ensure anonymity and rigor $(n=14)$, an indicative title containing information that it was a qualitative study $(n=13)$, the characteristics of the data collector that may influence the research such as qualification and relationship with participants $(n=12)$, and the context from where participants were recruited $(n=12)$.

All 6 studies that included a questionnaire were missing at least $40 \%$ of the items considered important on the Tong et al [28] 16-item checklist for reporting questionnaire studies (Multimedia Appendix 3). None of the 6 studies included details on participants' response rate, characteristics of refusals, if follow-up reminder was provided, and whether the questionnaire was piloted.

Of the 8 studies that reported an intervention efficacy trial, 3 were missing at least $40 \%$ of the items considered important on the D\&B checklist (Multimedia Appendix 4). All the studies did not detail how the sample size was estimated, a majority did not detail if blinding of participants and data collectors occurred $(n=7)$ and if there were any adverse events as a result of the intervention or the lack of it $(n=7)$. In 5 studies, the reporting of the results was not ideal $[19,37,41,44,58]$. For example, 3 studies reported within-group comparisons, whereas the reporting of between-group comparisons across time was lacking in detail $[19,37,44]$. One study reported having the intervention group showing trends for "better diabetes self-efficacy, better general treatment and less perceived stress" [58, p.7] than the control group; however, the reported $P$ values were .20 , thereby, demonstrating a lack of statistical significance.

\section{Summary of Interventions $(\mathbf{n}=\mathbf{2 2})$}

Of the 22 studies which provided a description of the intervention (Multimedia Appendix 5), 8 were evaluated in an efficacy trial. All of the 8 evaluations were conducted on Web-based interventions $[19,37,41,44,48,56,58]$.

Most interventions were delivered via a website $(n=13)$ $[19,34,37,38,41,42,44,46,48,49,52,55,56,58]$. Other modes of delivery included a mobile app $(\mathrm{n}=4)[47,50,57,59]$ and a choice of 3 websites and 3 mobile apps developed by peers $(n=1)$ [36]. In addition, 8 interventions were based upon theories, such as Social Cognitive Theory, Self-Determination Theory, Self-Efficacy Theory, and Social Learning Theory $[19,33,44,46,47,49,57,58]$.

\section{Adolescents and Young Adults' Perceived Usefulness or Acceptability $(n=16)$}

Perceived usefulness or acceptability was explored in 14 interventions across 16 studies because 2 of the interventions, Challenge your arthritis [33,34] and Teens Taking Charge: Managing Arthritis Online [55,56] were evaluated twice (Multimedia Appendix 6). Most of the interventions $(n=11)$ received a positive reaction from the participants $[33,34,37,42,44,46,47,51,52,55-58]$. Of the 3 interventions that received a neutral response, 2 did not involve pediatric patients in the intervention development [50,59], whereas one fully relied on AYAs without the involvement of a health care professional or content expert [36].

\section{Adolescents and Young Adults' Preferred Intervention Design}

The use of preexisting technology, such as a mobile app, not designed specifically for AYAs was not appealing to them [50]. As indicated by $91 \%$ of AYAs in the study conducted by Ashurst et al [36], AYAs' input was important to ensure that the intervention was tailored to their needs. 


\section{Preferred Delivery Method}

AYAs' preferred delivery method ranged from concise text to interactive content $[50,53,58]$. Placement of the information was important, whereby one study reported that AYAs wanted the most important information on each page at the top of the page [55]. Adolescents suggested incorporating visually appealing features, such as pictures and graphics [55], and games or audiovisual medium to create an engaging website $[42,43,51,54]$. Learning about medications through interactive games on a tablet or watching educational videos at a kiosk was also preferred [32]. Quizzes were considered more engaging than the sole use of text [54]. In addition, visual aids that allowed patients to view the severity level of their condition based on their symptoms were found be useful [50].

The main advantage of a Web- or mobile-based intervention was that it was readily accessible and it could be browsed at any time at the AYA's preferred pace $[38,39]$. To optimize uptake, AYAs suggested that a mobile app should be affordable and be made available on major mobile platforms whilst a website should be mobile-optimized [53]. Furthermore, an app that could perform offline would further optimize usage [51].

Short videos were very appealing to AYAs, especially those that provide support on the medical, lifestyle, and psychological aspects of living with a chronic disease [50,52]. Video testimonial by young people was deemed as an important way for AYAs to realize that they were not alone in their struggles [38]. In those interventions that incorporated videos or life stories, AYAs were able to understand the content easily and enjoyed watching the videos because they recognized themselves in the stories relayed $[34,42,52]$.

\section{Information Needs}

AYAs suggested using peers with chronic disease to comment on topics such as disease management tips, transition experiences [39], and disease experiences [54]. In addition, AYAs would like to receive disease-specific news or research updates $[39,54]$. In preparation for their transition to adult care, AYAs sought to obtain practical information, such as the differences between child and adult health care, the clinic's location, and key staff members [38].

Across different conditions, mental health support was found to be an appreciated feature by AYAs $[34,39,47]$. Huang et al [39] reported that young adults wanted to learn how to manage anxiety and stress, intimate relationships, alcohol and drug situations, and health insurance regardless of their condition. Similarly, Ammerlaan et al [34] reported that the most appealing topics were on how to deal with pain, fatigue and emotions, physical exercise, holidays, study, and work. Hence, rather than emphasizing health care and medical information, AYAs preferred to learn about challenges at school, work, and social settings and the emotional burden that they would be experiencing [49].

Suggestions to include disease-specific information were mentioned by AYAs. For example, patients with asthma mentioned about incorporating real-time reports concerning environmental conditions, such as pollen levels and weather forecasts, within an app [50]. They wanted a mobile app that could be customized to include their own profile with a personal medical history and treatment summary, reminders, a symptom tracker, access to emergency information even in the absence of an internet connection, and motivational feedback [47,51]. Conversely, young adults with juvenile idiopathic arthritis wanted reliable sources of medication information to be accessible via the website [34]. For organ transplant recipients, they wanted to have information relevant to the pretransplant period, details about medical emergencies or complications, and a section for parents [42]. Patients with hemophilia sought to learn about the pathology and severity of hemophilia, first aid, emergency services, and activity limitations [54].

\section{Support From Peers or Health Care Professionals}

Compared with face-to-face interactions, AYAs preferred an online support group [40,54]. One study reported that AYAs were wary of using chat rooms and existing social media sites to talk to strangers because of privacy concerns [35]. However, 7 other studies reported that AYAs would like to have the opportunity to network with their peers [34,39,42,43,53-55], which could help to ease feelings of isolation. Online discussion forums [34,42,54] or question and answer forums or use of a platform to share achievements [47] were suggested by AYAs as ways that they could feel connected with others diagnosed with the same condition. Although they might not post to the forum, $96 \%$ of AYAs revealed that reading comments posted by others was useful [45]. Some AYAs liked being able to contact or share information with their health care providers $[35,47,50,51]$.

\section{Synthesis of Quantitative Data $(n=8)$}

A total of 8 studies included an intervention efficacy trial, and all evaluations were conducted on Web-based interventions; none included a mobile app (Multimedia Appendix 7). For one study that had 3 groups of participants, the evaluation of intervention efficacy was reported based on the comparison between the "standardized patient management program (SPMP)" group and the "internet-based education program plus standardized patient management program (IEP+SPMP)" group as opposed to the "usual care" group [48]. Meta-analysis did not include studies that had no control group [46] and those that did not report standard deviation $[48,58]$ as group comparisons could not be conducted. Information requests were sent but there was no response from the corresponding authors $[48,58]$.

The most frequently measured outcomes were quality of life, self-efficacy, and self-management. The combined data for meta-analysis showed that there was no statistically significant group difference in quality of life $(\mathrm{n}=3$, standardized mean difference $-0.15,95 \% \mathrm{CI}-0.52$ to $0.22 ; P=.43$ ), self-efficacy $(\mathrm{n}=3$, standardized mean difference $0.15,95 \% \mathrm{CI}-0.17$ to 0.47 ; $P=.23)$, and self-management $(n=3$, standardized mean difference $0.11,95 \% \mathrm{CI}-0.18$ to $0.40 ; P=.44)$.

\section{Discussion}

\section{Principal Findings}

This systematic review examined 29 articles, which were published between 2006 and 2019, and included primary 
research articles discussing Web- and mobile-based interventions.

Using the qualitative data, this systematic review revealed that AYAs were very receptive to obtaining information electronically. AYAs were more likely to perceive an intervention as useful when there was a concerted effort of involving AYAs and experts in the process of intervention design as opposed to relying solely on AYAs or experts alone. However, engaging AYAs in research could be difficult. Ashurst et al [36] reported that many AYAs cited study commitment as a reason for nonparticipation or withdrawal although the project was conducted at a time that coincided with school holidays. Similarly, Simmons et al [52] faced difficulties in recruiting participants although an extensive strategy was used, and a cash incentive was offered. With the growing consensus about the crucial role that patients play in improving the value of health care research, there is a clear need to identify the best methods to achieve engagement, which is currently lacking [61]. To engage AYAs, research suggests that the most effective recruitment approach may be one that is initiated by AYAs' own health care providers combined with social media outreach and frequent contact [62].

Although AYAs had different preferred styles of message delivery, ranging from static text to audiovisual materials, being concise was the most important part to keeping them engaged. The findings of this systematic review revealed approaches that can be undertaken to design an intervention for AYAs; however, it also contains suggestions suitable for young school-aged children. The use of engaging technology can be a fun and easy way to captivate patients' attention and to encourage learning [32,35,53]. For example, young school-aged children can benefit from an intervention with audiovisual content about self-management and their condition. However, depending on the age group that the intervention is targeting, there is a need to use age-appropriate language.

Using the combined quantitative data, the meta-analysis showed that efficacy of the interventions on quality of life, self-efficacy, and self-management skills could not be found. With only 3 sets of data available for each outcome, the insignificant findings could be because of the study heterogeneity $\left(\mathrm{I}^{2}\right.$ score ranged from $27 \%$ for self-management to $50 \%$ for quality of life). A conclusion cannot be drawn on the overall effect of a Web-based intervention in preparing AYAs to self-manage their condition and to become independent adults in comparison with usual care. As there was no intervention efficacy trial on a mobile app, no conclusion can be drawn if a mobile app is a better tool for AYAs than a website. There was also a lack of high-quality randomized controlled trials as only 3 intervention efficacy trials obtained a D\&B score of $\geq 20$ out of a possible 27 items. The omission of information in intervention efficacy reports made it hard to assess if there were biases that could have influenced the findings. In particular, the reports did not detail the characteristics of participants who were lost to follow-up, and if blinding of participant and data collector was achieved. Similarly, studies that utilized a questionnaire failed to detail response rates and if the questionnaire used was piloted before distribution. The methodological shortcomings make it difficult for future investigators to adopt or refine the strategies used in designing or refining an intervention.

To date, systematic reviews have been conducted to examine the use of technology-based interventions in young people, such as the prevention and treatment of pediatric obesity [63] and suicide prevention [64]. Like the findings of this systematic review, other researchers found that there is a paucity of current evidence for technology-based interventions to improve patient outcomes [63,64]. The use of a Web- or mobile-based intervention is a relatively new area. As evidenced in this systematic review, the oldest study retrieved was published in 2006. There is a rise in the number of research studies undertaken given that most studies $(n=17,61 \%)$ were published between 2015 and 2019. Although there are many trials exploring AYAs' perspectives, quality randomized controlled trials assessing the efficacy of an intervention are lacking.

\section{Limitations}

The findings of this systematic review and meta-analysis should be interpreted with caution because of several methodological limitations. First, in the hope of uncovering replicable and inexpensive interventions, this systematic review focused on interventions that did not require extra resources, such as a device or a third party. However, it was found that AYAs wanted to obtain support from their peers or health professionals online. The extent of how this support enhanced the effectiveness and the reach of an intervention could not be concluded from this systematic review. Second, the included studies consisted of qualitative, quantitative, and mixed-methods studies, making it difficult to compare the quality of the studies. The different study designs also meant that the data were so varied that it was difficult to ascertain the effects of the interventions on overall AYAs' transition readiness. Nonetheless, the findings provided information on AYAs' preferred intervention design for future work. Finally, although there were no language restrictions when searching the major medical databases, it was acknowledged that a search including foreign language databases may reveal additional studies published in languages other than English in developed and developing countries.

\section{Conclusions}

This systematic review revealed that AYAs were receptive to receiving information through a website or mobile app, which is a first step to engaging them in their own care. Although no conclusion can be drawn on an effective intervention design because of the lack of intervention efficacy trials, this systematic review contained information about AYAs' preferred intervention. In designing an AYA-focused intervention, the best approach would be to first identify AYAs' disease-specific needs. This is to be coupled with or followed by obtaining suggestions from health professionals caring for AYAs. Finally, it is essential to obtain AYAs' feedback on the style and content of the designed intervention. Such a systematic iterative process will ensure that the designed intervention is accepted by AYAs, in the hope that it will improve patient engagement during the transition process and, thus, patient outcomes. Providing AYAs an age-appropriate, reliable condition-specific resource, which can be accessed anywhere, is the very first step in supporting 
them to becoming resourceful independent adults managing their own care.

\section{Acknowledgments}

This systematic review was funded by a Dean's Research Postdoctoral Fellowship Project Grant from Deakin University, Australia. The authors thank the librarian, Louisa Sher, for helping to set up the search strategy.

\section{Authors' Contributions}

JKL and EM were responsible for the idea conception, protocol refinement, and data analysis. JKL contributed to the drafting, whereas EM contributed to the revision of the manuscript.

\section{Conflicts of Interest}

None declared.

\section{Multimedia Appendix 1}

Search strategy.

[PDF File (Adobe PDF File), 302KB-Multimedia Appendix 1]

\section{Multimedia Appendix 2}

Quality appraisal of the qualitative component of the studies.

[PDF File (Adobe PDF File), 303KB-Multimedia Appendix 2]

\section{Multimedia Appendix 3}

Quality appraisal of the questionnaire component of the studies.

[PDF File (Adobe PDF File), 336KB-Multimedia Appendix 3]

\section{Multimedia Appendix 4}

Quality appraisal of intervention efficacy trials.

[PDF File (Adobe PDF File), 351KB-Multimedia Appendix 4]

\section{Multimedia Appendix 5}

Characteristics of included interventions.

[PDF File (Adobe PDF File), 132KB-Multimedia Appendix 5]

\section{Multimedia Appendix 6}

A summary of feasibility study or users' perceived acceptance of theintervention $(n=16)$.

[PDF File (Adobe PDF File), 458KB-Multimedia Appendix 6]

\section{Multimedia Appendix 7}

A summary of intervention efficacy evaluations $(n=8)$.

[PDF File (Adobe PDF File), 762KB-Multimedia Appendix 7]

\section{References}

1. Dabelea D, Mayer-Davis EJ, Saydah S, Imperatore G, Linder B, Divers J, SEARCH for Diabetes in Youth Study. Prevalence of type 1 and type 2 diabetes among children and adolescents from 2001 to 2009. J Am Med Assoc 2014 May 7;311(17):1778-1786 [FREE Full text] [doi: 10.1001/jama.2014.3201] [Medline: 24794371]

2. Patterson CC, Gyürüs E, Rosenbauer J, Cinek O, Neu A, Schober E, et al. Trends in childhood type 1 diabetes incidence in Europe during 1989-2008: evidence of non-uniformity over time in rates of increase. Diabetologia 2012 Aug;55(8):2142-2147. [doi: 10.1007/s00125-012-2571-8] [Medline: 22638547]

3. Anandan C, Nurmatov U, van Schayck OC, Sheikh A. Is the prevalence of asthma declining? Systematic review of epidemiological studies. Allergy 2010 Feb;65(2):152-167. [doi: 10.1111/j.1398-9995.2009.02244.x] [Medline: 19912154] 
4. Siegel RL, Miller KD, Jemal A. Cancer statistics, 2016. CA Cancer J Clin 2016;66(1):7-30 [FREE Full text] [doi: 10.3322/caac.21332] [Medline: 26742998]

5. Quinn CT, Rogers ZR, McCavit TL, Buchanan GR. Improved survival of children and adolescents with sickle cell disease. Blood 2010 Apr 29;115(17):3447-3452 [FREE Full text] [doi: 10.1182/blood-2009-07-233700] [Medline: 20194891]

6. Blum RW, Garell D, Hodgman CH, Jorissen TW, Okinow NA, Orr DP, et al. Transition from child-centered to adult health-care systems for adolescents with chronic conditions. A position paper of the society for adolescent medicine. $\mathrm{J}$ Adolesc Health 1993 Nov;14(7):570-576. [doi: 10.1016/1054-139X(93)90143-D] [Medline: 8312295]

7. Lugasi T, Achille M, Stevenson M. Patients' perspective on factors that facilitate transition from child-centered to adult-centered health care: a theory integrated metasummary of quantitative and qualitative studies. J Adolesc Health 2011 May;48(5):429-440. [doi: 10.1016/j.jadohealth.2010.10.016] [Medline: 21501800]

8. Sheehan AM, While AE, Coyne I. The experiences and impact of transition from child to adult healthcare services for young people with type 1 diabetes: a systematic review. Diabet Med 2015 Apr;32(4):440-458. [doi: 10.1111/dme.12639] [Medline: 25407592]

9. Hemker BG, Brousseau DC, Yan K, Hoffmann RG, Panepinto JA. When children with sickle-cell disease become adults: lack of outpatient care leads to increased use of the emergency department. Am J Hematol 2011 Oct;86(10):863-865 [FREE Full text] [doi: 10.1002/ajh.22106] [Medline: 21815184]

10. Lotstein DS, Seid M, Klingensmith G, Case D, Lawrence JM, Pihoker C, SEARCH for Diabetes in Youth Study Group. Transition from pediatric to adult care for youth diagnosed with type 1 diabetes in adolescence. Pediatrics 2013 Apr;131(4):e1062-e1070 [FREE Full text] [doi: 10.1542/peds.2012-1450] [Medline: 23530167]

11. Kipps S, Bahu T, Ong K, Ackland FM, Brown RS, Fox CT, et al. Current methods of transfer of young people with type 1 diabetes to adult services. Diabet Med 2002 Aug;19(8):649-654. [doi: 10.1046/j.1464-5491.2002.00757.x] [Medline: 12147145]

12. Heery E, Sheehan AM, While AE, Coyne I. Experiences and outcomes of transition from pediatric to adult health care services for young people with congenital heart disease: a systematic review. Congenit Heart Dis 2015;10(5):413-427. [doi: 10.1111/chd.12251] [Medline: 25659600]

13. Cole R, Ashok D, Razack A, Azaz A, Sebastian S. Evaluation of outcomes in adolescent inflammatory bowel disease patients following transfer from pediatric to adult health care services: case for transition. J Adolesc Health 2015 Aug;57(2):212-217. [doi: 10.1016/j.jadohealth.2015.04.012] [Medline: 26206442]

14. Annunziato RA, Emre S, Shneider B, Barton C, Dugan CA, Shemesh E. Adherence and medical outcomes in pediatric liver transplant recipients who transition to adult services. Pediatr Transplant 2007 Sep;11(6):608-614. [doi: 10.1111/j.1399-3046.2007.00689.x] [Medline: 17663682$]$

15. McDonagh JE, Southwood TR, Shaw KL, British Society of Paediatric and Adolescent Rheumatology. The impact of a coordinated transitional care programme on adolescents with juvenile idiopathic arthritis. Rheumatology (Oxford) 2007 Jan;46(1):161-168. [doi: 10.1093/rheumatology/kel198] [Medline: 16790451]

16. Watson AR. Problems and pitfalls of transition from paediatric to adult renal care. Pediatr Nephrol 2005 Feb;20(2):113-117. [doi: 10.1007/s00467-004-1763-y] [Medline: 15627164]

17. Campbell F, Biggs K, Aldiss SK, O'Neill PM, Clowes M, McDonagh J, et al. Transition of care for adolescents from paediatric services to adult health services. Cochrane Database Syst Rev 2016 Apr 29;4:CD009794. [doi: 10.1002/14651858.CD009794.pub2] [Medline: 27128768]

18. Betz CL, Smith K, Macias K. Testing the transition preparation training program: a randomized controlled trial. Int J Child Adolesc health 2010;3(4):595-607. [Medline: 22229060]

19. Huang JS, Terrones L, Tompane T, Dillon L, Pian M, Gottschalk M, et al. Preparing adolescents with chronic disease for transition to adult care: a technology program. Pediatrics 2014 Jun;133(6):e1639-e1646 [FREE Full text] [doi: 10.1542/peds.2013-2830] [Medline: 24843066]

20. Mackie AS, Islam S, Magill-Evans J, Rankin KN, Robert C, Schuh M, et al. Healthcare transition for youth with heart disease: a clinical trial. Heart 2014 Jul;100(14):1113-1118. [doi: 10.1136/heartjnl-2014-305748] [Medline: 24842870]

21. Steinbeck KS, Shrewsbury VA, Harvey V, Mikler K, Donaghue KC, Craig ME, et al. A pilot randomized controlled trial of a post-discharge program to support emerging adults with type 1 diabetes mellitus transition from pediatric to adult care. Pediatr Diabetes 2015 Dec;16(8):634-639. [doi: 10.1111/pedi.12229] [Medline: 25385685]

22. Stinson J, Wilson R, Gill N, Yamada J, Holt J. A systematic review of internet-based self-management interventions for youth with health conditions. J Pediatr Psychol 2009 Jun;34(5):495-510. [doi: 10.1093/jpepsy/jsn115] [Medline: 19029142]

23. Charlier N, Zupancic N, Fieuws S, Denhaerynck K, Zaman B, Moons P. Serious games for improving knowledge and self-management in young people with chronic conditions: a systematic review and meta-analysis. J Am Med Inform Assoc 2016 Jan;23(1):230-239. [doi: 10.1093/jamia/ocv100] [Medline: 26186934]

24. Low JK, Manias E. Centre for Reviews and Dissemination - University of York. 2018. Use of Technology-Based Interventions to Aid Transition in Adolescents and Young Adults With Chronic Disease: Protocol for a Systematic Review of Qualitative and Quantitative Studies URL: http://www.crd.york.ac.uk/PROSPERO/display record.php?ID=CRD42018096487 [accessed 2019-04-26] [WebCite Cache ID 77zBxUuPd] 
25. Moher D, Liberati A, Tetzlaff J, Altman DG, PRISMA Group. Preferred reporting items for systematic reviews and meta-analyses: the PRISMA statement. PLoS Med 2009 Jul 21;6(7):e1000097. [doi: 10.1371/journal.pmed.1000097] [Medline: 19621072]

26. Downs SH, Black N. The feasibility of creating a checklist for the assessment of the methodological quality both of randomised and non-randomised studies of health care interventions. J Epidemiol Community Health 1998 Jun;52(6):377-384 [FREE Full text] [doi: 10.1136/jech.52.6.377] [Medline: 9764259]

27. O'Brien BC, Harris IB, Beckman TJ, Reed DA, Cook DA. Standards for reporting qualitative research: a synthesis of recommendations. Acad Med 2014 Sep;89(9):1245-1251 [FREE Full text] [doi: 10.1097/ACM.0000000000000388] [Medline: 24979285]

28. Tong A, Wong G, Hodson E, Walker RG, Tjaden L, Craig JC. Adolescent views on transition in diabetes and nephrology. Eur J Pediatr 2013 Mar;172(3):293-304. [doi: 10.1007/s00431-012-1725-5] [Medline: 22576804]

29. Trac MH, McArthur E, Jandoc R, Dixon SN, Nash DM, Hackam DG, et al. Macrolide antibiotics and the risk of ventricular arrhythmia in older adults. Can Med Assoc J 2016 Apr 19;188(7):E120-E129 [FREE Full text] [doi: 10.1503/cmaj.150901] [Medline: 26903359]

30. Stuber KJ, Smith DL. Chiropractic treatment of pregnancy-related low back pain: a systematic review of the evidence. J Manipulative Physiol Ther 2008;31(6):447-454. [doi: 10.1016/j.jmpt.2008.06.009] [Medline: 18722200]

31. Thomas J, Harden A. Methods for the thematic synthesis of qualitative research in systematic reviews. BMC Med Res Methodol 2008 Jul 10;8:45 [FREE Full text] [doi: 10.1186/1471-2288-8-45] [Medline: 18616818]

32. Abraham O, Brothers A, Alexander DS, Carpenter DM. Pediatric medication use experiences and patient counseling in community pharmacies: perspectives of children and parents. J Am Pharm Assoc (2003) 2017;57(1):38-46.e2 [FREE Full text] [doi: 10.1016/j.japh.2016.08.019] [Medline: 27843107]

33. Ammerlaan J, van Os-Medendorp H, Scholtus L, de Vos A, Zwier M, Bijlsma H, et al. Feasibility of an online and a face-to-face version of a self-management program for young adults with a rheumatic disease: experiences of young adults and peer leaders. Pediatr Rheumatol Online J 2014 Mar 25;12:10 [FREE Full text] [doi: 10.1186/1546-0096-12-10] [Medline: 24666817]

34. Ammerlaan JJ, Scholtus LW, Drossaert CH, van Os-Medendorp H, Prakken B, Kruize AA, et al. Feasibility of a website and a hospital-based online portal for young adults with juvenile idiopathic arthritis: views and experiences of patients. JMIR Res Protoc 2015 Aug 14;4(3):e102 [FREE Full text] [doi: 10.2196/resprot.3952] [Medline: 26276373]

35. Applebaum MA, Lawson EF, von Scheven E. Perception of transition readiness and preferences for use of technology in transition programs: teens' ideas for the future. Int J Adolesc Med Health 2013;25(2):119-125. [doi: 10.1515/ijamh-2013-0019] [Medline: 23740658]

36. Ashurst EJ, Jones RB, Abraham C, Jenner M, Boddy K, Besser RE, et al. The diabetes app challenge: user-led development and piloting of internet applications enabling young people with diabetes to set the focus for their diabetes consultations. Med 202014 Nov 7;3(2):e5 [FREE Full text] [doi: 10.2196/med20.3032] [Medline: 25654312]

37. Breakey VR, Ignas DM, Warias AV, White M, Blanchette VS, Stinson JN. A pilot randomized control trial to evaluate the feasibility of an internet-based self-management and transitional care program for youth with haemophilia. Haemophilia 2014 Nov;20(6):784-793. [doi: 10.1111/hae.12488] [Medline: 25311370]

38. Coyne I, Prizeman G, Sheehan A, Malone H, While AE. An e-health intervention to support the transition of young people with long-term illnesses to adult healthcare services: design and early use. Patient Educ Couns 2016 Dec;99(9):1496-1504. [doi: 10.1016/j.pec.2016.06.005] [Medline: 27372524]

39. Huang JS, Gottschalk M, Pian M, Dillon L, Barajas D, Bartholomew LK. Transition to adult care: systematic assessment of adolescents with chronic illnesses and their medical teams. J Pediatr 2011 Dec;159(6):994-8.e2 [FREE Full text] [doi: 10.1016/j.jpeds.2011.05.038] [Medline: 21784450]

40. Johnson KR, Fuchs E, Horvath KJ, Scal P. Distressed and looking for help: internet intervention support for arthritis self-management. J Adolesc Health 2015 Jun;56(6):666-671. [doi: 10.1016/j.jadohealth.2015.02.019] [Medline: 26003583]

41. Joseph CL, Peterson E, Havstad S, Johnson CC, Hoerauf S, Stringer S, Asthma in Adolescents Research Team. A web-based, tailored asthma management program for urban African-American high school students. Am J Respir Crit Care Med 2007 May 1;175(9):888-895 [FREE Full text] [doi: 10.1164/rccm.200608-12440C] [Medline: 17290041]

42. Korus M, Cruchley E, Stinson JN, Gold A, Anthony SJ. Usability testing of the internet program: 'teens taking charge: managing my transplant online'. Pediatr Transplant 2015 Feb;19(1):107-117. [doi: 10.1111/petr.12396] [Medline: 25495484]

43. Lopez KN, O'Connor M, King J, Alexander J, Challman M, Lovick DK, et al. Improving transitions of care for young adults with congenital heart disease: mobile app development using formative research. JMIR Form Res 2018;2(2):pii: e16 [FREE Full text] [doi: 10.2196/formative.9963] [Medline: 30574573]

44. Mulvaney SA, Rothman RL, Wallston KA, Lybarger C, Dietrich MS. An internet-based program to improve self-management in adolescents with type 1 diabetes. Diabetes Care 2010 Mar;33(3):602-604 [FREE Full text] [doi: 10.2337/dc09-1881] [Medline: 20032275]

45. Mulvaney SA, Rothman RL, Osborn CY, Lybarger C, Dietrich MS, Wallston KA. Self-management problem solving for adolescents with type 1 diabetes: intervention processes associated with an internet program. Patient Educ Couns 2011 Nov;85(2):140-142 [FREE Full text] [doi: 10.1016/j.pec.2010.09.018] [Medline: 21030194] 
46. Paul LL. ProQuest. 2017. Can-do-tude: An Online Intervention Using Principles of Motivational Interviewing and Tailored Diabetes Self-Management Education for Adolescents With Type 1 Diabetes URL: https://search.proquest.com/docview/ 1944399503 [accessed 2018-05-17] [WebCite Cache ID 71tsBPi0u]

47. Peters D, Davis S, Calvo RA, Sawyer SM, Smith L, Foster JM. Young people's preferences for an asthma self-management app highlight psychological needs: a participatory study. J Med Internet Res 2017 Dec 11;19(4):e113 [FREE Full text] [doi: 10.2196/jmir.6994] [Medline: 28400353]

48. Runge C, Lecheler J, Horn M, Tews JT, Schaefer M. Outcomes of a web-based patient education program for asthmatic children and adolescents. Chest 2006 Mar;129(3):581-593. [doi: 10.1378/chest.129.3.581] [Medline: 16537855]

49. Scal PB, Garwick AE, Horvath KJ. Making rheumtogrow: the rationale and framework for an internet based health care transition intervention. Int J Child Adolesc Health 2010;3(4):451-461 [FREE Full text]

50. Schneider T, Panzera AD, Couluris M, Lindenberger J, McDermott R, Bryant CA. Engaging teens with asthma in designing a patient-centered mobile app to aid disease self-management. Telemed J E Health 2016 Feb;22(2):170-175. [doi: 10.1089/tmj.2015.0041] [Medline: 26258994]

51. Schneider T, Baum L, Amy A, Marisa C. I have most of my asthma under control and I know how my asthma acts: Users' perceptions of asthma self-management mobile app tailored for adolescents. Health Informatics J 2019 Feb 08 (forthcoming). [doi: 10.1177/1460458218824734] [Medline: 30732520]

52. Simmons GM, Frick N, Wang A, Miller ME, Fragueiro D. Identifying information needs among children and teens living with haemophilia. Haemophilia 2014 Jan;20(1):1-8 [FREE Full text] [doi: 10.1111/hae.12223] [Medline: 23809876]

53. Slater H, Jordan JE, Chua J, Schütze R, Wark JD, Briggs AM. Young people's experiences of persistent musculoskeletal pain, needs, gaps and perceptions about the role of digital technologies to support their co-care: a qualitative study. BMJ Open 2016 Dec 9;6(12):e014007 [FREE Full text] [doi: 10.1136/bmjopen-2016-014007] [Medline: 27940635]

54. Sterling L, Nyhof-Young J, Blanchette VS, Breakey VR. Exploring internet needs and use among adolescents with haemophilia: a website development project. Haemophilia 2012 Mar;18(2):216-221. [doi: 10.1111/j.1365-2516.2011.02613.x] [Medline: 21797947]

55. Stinson J, McGrath P, Hodnett E, Feldman B, Duffy C, Huber A, et al. Usability testing of an online self-management program for adolescents with juvenile idiopathic arthritis. J Med Internet Res 2010 Jul 29;12(3):e30 [FREE Full text] [doi: 10.2196/jmir.1349] [Medline: 20675293]

56. Stinson JN, McGrath PJ, Hodnett ED, Feldman BM, Duffy CM, Huber AM, et al. An internet-based self-management program with telephone support for adolescents with arthritis: a pilot randomized controlled trial. J Rheumatol 2010 Sep;37(9):1944-1952. [doi: 10.3899/jrheum.091327] [Medline: 20595280]

57. Stinson JN, Lalloo C, Harris L, Isaac L, Campbell F, Brown S, et al. iCanCope with pain ${ }^{\mathrm{TM}}$ : user-centred design of a weband mobile-based self-management program for youth with chronic pain based on identified health care needs. Pain Res Manag 2014;19(5):257-265 [FREE Full text] [doi: 10.1155/2014/935278] [Medline: 25000507]

58. Whittemore R, Grey M, Lindemann E, Ambrosino J, Jaser S. Development of an internet coping skills training program for teenagers with type 1 diabetes. Comput Inform Nurs 2010;28(2):103-111 [FREE Full text] [doi: 10.1097/NCN.0b013e3181cd8199] [Medline: 20182161]

59. Zhao Y, Calvo R, Pardo A, Gunn H, Steinbeck K. What We Learned From TransitionMate: A Mobile App Designed to Support Young People With Chronic Illness. In: Proceedings of the Annual Meeting of the Australian Special Interest Group for Computer Human Interaction. 2015 Presented at: OzCHI'15; December 7-10, 2015; Parkville, VIC, Australia p. 162-166. [doi: 10.1145/2838739.2838805]

60. Secor-Turner M, Scal P, Garwick A, Horvath K, Wells CK. Living with juvenile arthritis: adolescents' challenges and experiences. J Pediatr Health Care 2011;25(5):302-307. [doi: 10.1016/j.pedhc.2010.06.004] [Medline: 21867858]

61. Domecq JP, Prutsky G, Elraiyah T, Wang Z, Nabhan M, Shippee N, et al. Patient engagement in research: a systematic review. BMC Health Serv Res 2014 Feb 26;14:89. [doi: 10.1186/1472-6963-14-89] [Medline: 24568690]

62. Howarth S. Texas Christian University Digital Repository. 2017. Recruitment Challenges in Adolescent \& Young Adult Cancer Survivors URL: https://repository.tcu.edu/bitstream/handle/116099117/19833/Howarth_Sara-Honors_Project. pdf? sequence $=1$ [WebCite Cache ID 71 tqqPZKK]

63. Turner T, Spruijt-Metz D, Wen CK, Hingle MD. Prevention and treatment of pediatric obesity using mobile and wireless technologies: a systematic review. Pediatr Obes 2015 Dec;10(6):403-409 [FREE Full text] [doi: 10.1111/ijpo.12002] [Medline: 25641770]

64. Perry Y, Werner-Seidler A, Calear AL, Christensen H. Web-based and mobile suicide prevention interventions for young people: a systematic review. J Can Acad Child Adolesc Psychiatry 2016;25(2):73-79 [FREE Full text] [Medline: 27274742]

\section{Abbreviations}

AYAs: adolescents and young adults

D\&B: Downs and Black checklist

SRQR: Standards for Reporting Qualitative Research 
Edited by G Eysenbach; submitted 26.08.18; peer-reviewed by J Huang, S Davis; comments to author 14.10.18; revised version received 30.04.19; accepted 10.06.19; published 18.07.19

Please cite as:

Low JK, Manias E

Use of Technology-Based Tools to Support Adolescents and Young Adults With Chronic Disease: Systematic Review and Meta-Analysis JMIR Mhealth Uhealth 2019;7(7):e12042

URL: http://mhealth.jmir.org/2019/7/e12042/

doi: $10.2196 / 12042$

PMID: $\underline{31322129}$

(CJac Kee Low, Elizabeth Manias. Originally published in JMIR Mhealth and Uhealth (http://mhealth.jmir.org), 18.07.2019. This is an open-access article distributed under the terms of the Creative Commons Attribution License (https://creativecommons.org/licenses/by/4.0/), which permits unrestricted use, distribution, and reproduction in any medium, provided the original work, first published in JMIR mhealth and uhealth, is properly cited. The complete bibliographic information, a link to the original publication on http://mhealth.jmir.org/, as well as this copyright and license information must be included. 